\title{
Academic success: a student's guide to studying at university
}

A book review of Brick, J., Wilson, N., Wong, D., and Herke, M. (2019) Academic success: a student's guide to studying at university. London: Red Globe Press.

\section{Karen Hudson}

University of Essex, UK

Keywords: academic communication, culture and convention; knowledge construction; learning; reading; writing; critical thinking; academic voice.

\section{Overview}

Aimed at students with limited experience of the culture and conventions of Englishspeaking universities, this book introduces readers to a wide range of academic communicative practices. It assumes no prior knowledge or experience of the (mostly) unwritten behaviours, attitudes and values required for academic success and provides a comprehensive breakdown of these, clarified throughout with examples, explanations and practical guidance.

The book's scope is broad, rather than deep, and therefore represents a useful and pragmatic introductory text for any student preparing for a transition or return to higher education. The text is underpinned throughout by two recurring themes that are directly transferable into LD practice. The idea that academic knowledge is developed and communicated via debate and argument is directly linked to the notion of 'academic apprenticeship' in which students are encouraged to begin participation in active and current disciplinary discourse.

\section{Structure and Content}


The publication is divided into five overarching sections, each dealing with a particular subset of the key features of university study. Part one provides a general introduction to university study in English-speaking cultures. Chapter one introduces students to some academic conventions that are widely, perhaps implicitly, understood by academic staff, but which are not always explicitly communicated to new students. The chapter lays the foundation for one of the main underpinning concepts of the book: that academic knowledge is developed and communicated via debate and argument, agreement and disagreement, and that this frequently takes place via published texts. Students are introduced to the idea that dominant theories and ideas, both current and historical, are subject to challenge and development via this process, and that each discipline may have different communicative practices for this process. The basic features of academic argumentation are outlined with clear and practical examples, which provide a sound basis for further discussion in subsequent chapters.

Chapter two addresses generic issues of university organisation and structure, including titles, positions, roles and expectations of staff and students. It begins to prepare students for the variety of teaching and learning methods that they are likely to encounter, from lectures, to seminars, tutorials, and 'flipped classroom' methods. Due acknowledgement is given to the wide range of teaching philosophies that exist among $\mathrm{HE}$ practitioners, and potentially misguided student expectations with regards to knowledge transmission are well-managed here. 'Academic apprenticeship' provides a useful analogy for emphasising that students must not only acquire a body of knowledge; they must also develop the critical and analytical skills required to apply this knowledge in a wider context.

Chapter three focuses on independent learning, emphasising that in the English-speaking world people are considered independent adults from the age of 18 , and that this expectation is reflected in the university environment. Personal accountability is stressed throughout and the specific skills that underpin this are clearly indicated. Particularly useful here is the explicit listing of common areas of student difficulty in managing the transition to higher education (p.25), and practical guidance on how to resolve these. Some educators may find the section on learning styles frustrating; while it is acknowledged that there is little empirical evidence to support the alignment of teaching and learning styles, it may have been useful to suggest that, regardless of individual preferences, students may benefit from challenging and developing their capabilities across a wider range of learning resources. 
Having prepared readers for the structure and expectations of university learning in Part one, Part two introduces some practicalities of the university learning environment. Chapter four begins with an exploration of academic listening skills. Lectures are presented as one of four essential 'pillars' on which knowledge and understanding are built. The dependent relationship between lecture attendance and pro-active, independent learning is clearly articulated and students are reminded that lecturers will not provide all of the knowledge and critical understanding required for success. A return to the 'debate and argument' and 'academic apprenticeship' themes also serves to remind students that they will hear ideas in lectures that differ from those found in their reading material and vice versa, and that their role is to evaluate these in order to establish a position. LD practitioners who undertake classroom teaching as part of their role will find some useful tips here to develop engaging sessions and accessible, effective slides.

The next chapter explores academic reading, including common types of text, and an introduction to databases. With a further nod to the 'debate and argument' concept, the need to read multiple sources and arguments is reinforced. The 4-S (Searching, Skimming, Selecting, Studying) system is recommended, which appears to be generally sound, although perhaps lacking the questioning, review and recall elements of some other models. The technique of identifying and using topic sentences to construct a coherent outline of a topic is well-presented, and revisited in more detail in later chapters. A section on critical reading contains a useful line of questioning that clearly includes the need to compare your texts with the position of other writers in the field, although the supporting example on pp. 47-48 is perhaps a little unclear and potentially confusing for students at the beginning of their academic reading apprenticeship.

Chapter six deals with tutorials and seminars and seeks to address the common anxieties and misconceptions held by those new to academic study. Particularly welcome here is the reassurance that students are not expected to have a fully-formed, authoritative command of their topics in order to successfully participate in these sessions. Emphasis throughout is on active and independent preparation, reading, discussion and willingness to seek guidance from a range of academic sources. Here, we are all reminded that written academic English is no-one's native language, and a brief introduction to some key differences between spoken and written academic English is provided in an accessible table format. 
Group assignments are the focus of Chapter seven, and this book does not trivialise the frustrations frequently expressed towards this form of assessment. Nonetheless, the authors present a convincing argument for the graduate attributes that group work can develop, including basic project and people management skills, including culturally competent group interaction.

Part three introduces concepts of criticality and begins to unpack some of the academic vocabulary with which new students may be unfamiliar. Opinion, position and bias are explained, and students are introduced to both the everyday and academic meanings of these words. Careful attention is paid to the difference between personal opinion and academic position based on appropriate evidence. Chapter nine offers some very pragmatic responses to common student concerns, including how to respond to contradictory evidence and different scholarly positions. This section concludes with a comprehensive and detailed guide on how to evaluate sources, although a more specific definition of 'grey literature' (that which is not produced by traditional or identifiable publishers), and its place within academic discourse, would have been welcome here. Occasionally, some of the discussion of critical evaluation and analysis may be a little too ambitious for new students with a limited experience of academic convention; however, this is certainly a section that would bear repeated reading at a later stage.

Part four is dedicated to the use of a range of 'voices', including the student's own. Chapters eleven and twelve address the use of expert and student voice, identifying and explicitly naming features of academic writing that are frequently only tacitly known by academic staff. Pages 111-114 provide a sample piece of academic writing and accompanying commentary with external and internal voices clearly noted and their purpose explained and grounded in the 'debate and argument' concept that underpins the book. The text is very clear on the conventions and practices surrounding the use of voice, particularly that 'if a statement is not labelled with the names of the writers, it is taken to be the essay writer's voice'. This discussion lays a firm foundation for the introduction to citation, referencing and academic integrity that follows. Chapter thirteen ostensibly covers plagiarism and how to avoid it, although a wider range of academic offences are outlined here. Clear examples of plagiaristic and colluding behaviours are given, along with explanations of why these are unacceptable. The chapter focuses mainly on the underlying causes of what frequently appears to be unintentional plagiarism/poor 
academic practice, although more serious academic offences are also discussed. This section would have benefited from a discussion of how effective paraphrasing and summarising underpins academic authorship, and from encouraging students to become familiar with their own institutional policies and provision regarding academic integrity.

Chapter fourteen returns to the topic of the use and purpose of 'voice' in different types of academic texts. The different purposes of several text genres, including textbooks and journal articles, are introduced, and no assumptions are made that students will be familiar with these. The explicit clarification of different genres, along with their styles and features, are likely to prove helpful for students who are beginning to develop their academic reading and writing skills. Part four concludes with a more in-depth technical discussion of identity, voice and position that contains a careful and appropriate balance of both encouragement and caution. The authors remind students that their reading has a specific purpose and audience, noting that student writing, in general, needs to retain a formal, academic tone, even if this differs from the tone of their reading material.

Part five outlines the key features of successful student writing, building on the concepts discussed in previous chapters and linking back to the underpinning theme of knowledge construction via debate and discussion. Chapters sixteen and seventeen revisit academic apprenticeship, reassuring students that they will not be expected to write like a professional academic. However, they are reminded of the inevitable evaluation of their ability to use key academic writing concepts in their assessments. The familiar recursive process of pre-planning, task analysis, note-making, organisation, drafting, editing and proofreading is detailed here. Techniques for effective argumentation, including the use of topic sentences, clauses and word chains to develop coherent and cohesive paragraphs and arguments, are also outlined and explained. Chapter eighteen includes an interesting exploration of how deductive argumentation is highly valued in English-speaking universities, in contrast to the inductive reasoning methods that may be favoured in other educational cultures. A pragmatic approach is taken to this peculiarity; students are reminded again that their writing has both a purpose and an audience, and that successful writing aims to address lecturer expectations and specific learning outcomes. A sample annotated piece of writing on pp.177-179 places prior discussion of topic sentences, generalisations, specific evidence, a range of voices and position statements within a more complete context, and key features of effective writing are clearly presented without resorting to formulaic, rule-based approaches to structure. 
Chapters nineteen to twenty-three provide brief, yet insightful overviews of a wider range of the different assignment writing genres that students may encounter, including reports, research reports, reflective writing, blogs and exams. While it is not possible to cover any of these topics in real depth within the scope of this book, the basic requirements of each are outlined, and students are encouraged to think carefully about the use and relative dominance of a range of voices, and to take a critical and analytical approach to a range of problem-solving scenarios, and the making and evaluating of recommendations.

The book concludes with a comprehensive glossary of the key academic vocabulary used throughout, and suggestions for further reading, some of which, however, may be of more interest to academic development practitioners than to new students. It is supported by online resources, including a downloadable glossary, and a more extensive and detailed range of practical activities to support student learning.

\section{Summary}

Overall, this book represents a broad and pragmatic introduction to the fundamentals of university study, and of academic communication, culture and convention. It is very easy to navigate and is clear and accessible, both in the information that it delivers, and the activities designed to support them. It would make ideal pre-sessional reading for students who are not familiar with the practices of the English-speaking university, whether they are international or home students, and is a welcome addition to the study skills repertoire.

\section{Author details}

Karen Hudson is a Literacy, Language and Numeracy Tutor in the School of Health and Social Care at the University of Essex. 